\title{
Investigation of shock wave focusing in water in a logarithmic spiral duct, Part 1: Weak coupling
}

\author{
Chuanxi Wang ${ }^{\mathrm{a}}$, Shi Qiu ${ }^{\mathrm{b}}$, Veronica Eliasson ${ }^{\mathrm{b}, \mathrm{c}}$ \\ ${ }^{a}$ Department of Physics and Astronomy, University of Southern California, Los Angeles, \\ California 90089, USA \\ ${ }^{b}$ Department of Aerospace and Mechanical Engineering, University of Southern \\ California, Los Angeles, California 90089, USA \\ ${ }^{c}$ Email: eliasson@usc.edu, Phone: (213)740-7182, Fax: (213)740-7774
}

\section{Abstract}

Shock focusing in water is a phenomenon that can occur during the impact of a shock wave generated by an underwater explosion onto any type of convergent marine structures. To predict the dynamic material response of the marine structure, it is important to understand the shock wave dynamics during the focusing event. In this paper, both experimental investigations and numerical studies of two-dimensional shock focusing in water are presented. Here, a convergent geometry given by a logarithmic spiral curve is used to focus the shock waves. In the experiments, the interaction between three types of materials and the shock wave in water is explored by using high-speed photography. Distinct features of such flows are unveiled. Three scenarios have been considered in simulations; a rigid structure where only the water-filled region is taken into account, a fluid-structure interaction problem in which the surrounding material responses are included, and an axi-symmetric simulation to determine the three-dimensional effects. Results from the numerical simulations show that boundary conditions and initial conditions are important to accurately predict fluid properties during 
the shock focusing phase.

Keywords: Shock focusing, Underwater explosion, Fluid-structure interaction, Schlieren, Finite difference simulations

\section{Introduction}

2

Shock focusing is a fundamental tool to generate extreme conditions at the focal region. It is utilized in both basic research and various applications such as in the study of shock stability, implosion fusion reactors and medical treatment for kidney stones (Gardner et al., 1982; Lindl et al., 1992; Chaussy et al., 1980). Shock focusing typically occurs because of the reflection from complex boundaries surrounding the shock focusing media or inhomogeneities of the flow field (Sturtevant and Kulkarny, 1976). For example, a planar shock front becomes curved as it propagates inside a shock wave lithotripter, a reflector in a particular shape used to generate a focused shock wave to destroy kidney stones (Mulley, 1986). Many analytical, experimental and numerical studies have been conducted on shock focusing. Most of the work focused on the generation and stability of cylindrical and spherical converging shock waves in gases (Guderley, 1942; Perry and Kantrowitz, 1951; Takayama et al., 1987; Schwendeman and Whitham, 1987; Eliasson et al., 2008). In recent years, the use of shock wave focusing in biomedical applications has increased. Examples are shock wave lithotripsy (Mulley, 1986; Gerald, 1997; Weiland et al., 2007) and drug delivery by shock waves (Kato et al., 2000; Doukas and Kollias, 2004). The working medium for both of these applications is water or a water solution. In general, shock focusing in water generates much higher pressures than shock focusing in air, due to the 
increase in density and speed of sound in water as compared to air (Ridah, 1988; Fleck and Deshpande, 2004).

An application of particular interest to the navy is shock loading from underwater explosions (UNDEX). UNDEX poses a tremendous threat to all kinds of naval structures. In particular, shock wave focusing from UNDEX can take place in several convergent sections on a naval vessel, such as rudder-hull junction, propeller shaft and bow thruster. The main factor that influences the focusing behavior of the shock wave in water is the geometry of the convergent sections, as suggested by previous studies of shock focusing (Schwendeman and Whitham, 1987; Coleman and Saunders, 1989; Eliasson et al., 2007). Other factors, such as the shock strength and the material properties of the surrounding section, also play a critical role in determining the focusing effects of the shock wave.

In order to obtain an upper limit of the most severe conditions that UNDEX can generate, we propose to study shock focusing in geometric shapes leading to a maximized energy at the focal region. The geometric shape chosen for this study is given by a so-called logarithmic spiral which was presented first by Milton and Archer (Milton and Archer, 1969). Since the shape of the shock front far enough away from an UNDEX event can be approximated to be planar, all simulations in this paper starts with a planar incident shock wave.

Furthermore, multiphase interactions including liquid-gas interaction and liquid-solid interaction are common in shock focusing applications. For example, a study on superseismic loading shows that the fluid-solid interface deflection induced by the shock impact can result in modified fluid dynamics 
(Arienti and Shepherd, 2002). In general, multiphase interactions are challenging for both experimental and numerical investigations. In experiments, ultrafast diagnostic methods are needed in order to characterize the fluid and solid dynamics simultaneously (Espinosa et al., 2006; Giordano et al., 2005). In simulations, strategies to take the effects of coupling between the fluid and solid phase have been developed (Shin, 2004; Giordano et al., 2005; Young et al., 2009) and are available in many different simulation packages.

Here, we present results of shock focusing in a water-filled convergent section surrounded by a bulk material using both experiments and numerical simulations. The experiments consist of shock focusing in a water-filled convergent section surrounded by different types of bulk materials; polycarbonate, aluminum and polymethylmethacrylate/methacrylate (PMMA). The plastic materials are used because they are transparent and simultaneous wave propagation in the sample and inside the water-filled region can be obtained. The metallic sample is closer to the materials that are used in many marine applications. Three different types of simulations are presented and compared to experiments; (i) a two-dimensional rigid confinement, (ii) a two-dimensional multi-physics simulation that takes both the water in the convergent section and the surrounding solid material into account, and (iii) an axisymmetric rigid confinement. A two-dimensional rigid simulation is chosen because it should give the ideal maximum pressure at the focal region, providing an upper limit on e.g. maximum density and pressure close to the focal region, and it provides an understanding if the boundary can be assumed to be rigid or not. The two-dimensional fluid-structure simulation shows the importance of enabling energy from the incident shock wave to 
being distributed to the core material, as is the case in the experiments. It also shows how much the maximum pressure is reduced by assuming a linear elastic response of the surrounding structure. The last set of simulations, axi-symmetric, indicates the importance of taking three-dimensional effects into account.

\section{Experimental technique}

Experiments are performed using an impact technique where a projectile from a gas gun impacts onto the experimental specimen. The projectile impact leads to generation of a propagating shock wave in a water-filled convergent section in the experimental specimen. As the shock wave propagates into the convergent section it will focus, and generate very high pressures at the focal region. Two different schlieren visualization techniques are used to capture the shock dynamics and the interaction with the surrounding material during the focusing phase; single-shot photography with high resolution, and high-speed photography.

The experimental setup consist of a gas gun, a $25.4 \mathrm{~cm}$-diameter Z-folded schlieren imaging system, and the experimental specimen. The experimental technique and the visualization system have been explained elsewhere in detail (Wang and Eliasson, 2012; Wang et al., 2013), and will be only briefly explained here. The experimental specimen is shown in Figure 1. The core material with a convergent water-filled section is sandwiched between two transparent optical quality polycarbonate windows measuring $182 \times 205 \times 12.7 \mathrm{~mm}$. The shape of the convergent cavity is given by a logarithmic spiral and this shape was chosen due to its ability to focus a shock wave with minimal re- 
flections (Inoue et al., 1993, 1995). The shape is derived using Whitham's geometrical shock dynamic theory (Whitham, 1974) based on a shock Mach number $M_{s}=1.1$ with a stiffened equation of state (Wang and Eliasson, 2012). The length of the convergent cavity, also referred to as the characteristic length of the logarithmic spiral is set to $L=114 \mathrm{~mm}$, Figure 1 . A polycarbonate blocker is placed at the entrance of the convergent section to prevent the water from leaking out. The core materials are made of polycarbonate, aluminum or PMMA, and the windows are made of polycarbonate. The edges between the windows and the core are sealed with silicone to prevent water leakage from the specimen. Special care is taken to remove any bubbles in the water-filled region before an experiment. Details of the wave propagation through the blocker and the experimental specimen along with shock Hugoniot curves for the various materials involved are carefully explained in our earlier work (Wang and Eliasson, 2012).

\subsection{Single-shot photography}

High-resolution single-shot photographs are taken with a Nikon D90 SLR camera with a AF-S Nikkor 18-105 mm zoom lens using an 18 ns spark light source (Nanolite, High-Speed Photo-Systeme). The advantage of this technique is the high resolution, but only one photograph per test can be obtained. In the experiments presented in this paper, the camera is operating at $100 \mathrm{~mm}$ focal length with $2848 \times 4288$ pixel picture resolution, which corresponds to a $163 \mathrm{~mm} \times 245 \mathrm{~mm}$ region of the sample being visualized. During the experiments, the laboratory is in a dark room setting and the camera shutter will be open for two seconds waiting for a flash from the spark light source. When the projectile impacts onto the specimen, a trigger signal 


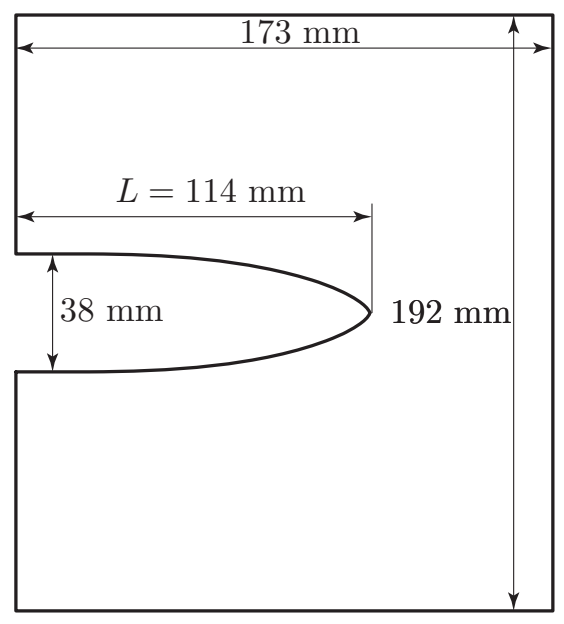

(a) Core

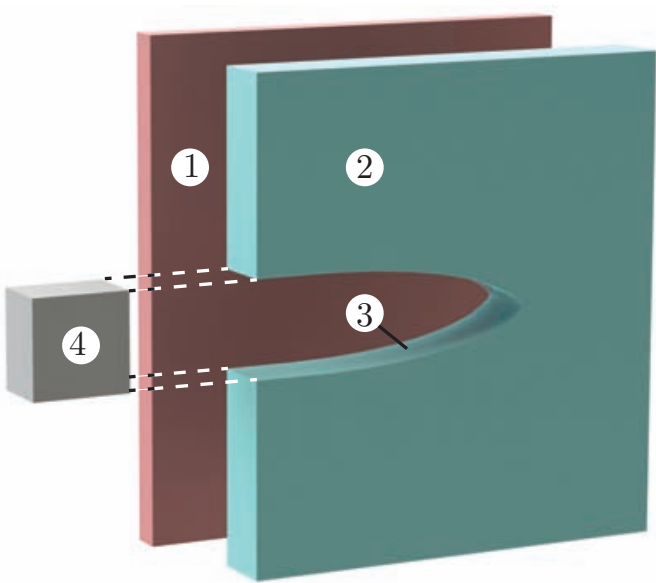

(b) Assembly

Figure 1: (a) Experimental core and exploded view of the assembly. The core thickness is $25.4 \mathrm{~mm}$ for the aluminum and polycarbonate samples. For the PMMA samples, this thickness is $6.3 \mathrm{~mm}$. (b) Exploded specimen assembly with (1) transparent window (only one is depicted for clarity), (2) core, (3) water-filled convergent section, and (4) blocker placed at the entrance of the cavity to serve as impact site and to prevent water from leaking out.

is sent out to a pulse/delay generator unit (BNC Model 575). The trigger signal comes from when the projectile breaks a laser beam just ahead of the blocker at the experimental specimen. After an appropriate time delay, the delay unit sends out a signal to trigger the spark source and the photograph can be recorded.

\subsection{High-speed photography}

A Phantom V711 camera, equipped with an AI-S Nikkor 50mm f/1.4 lens, is used for obtaining high-speed schlieren photos. All high-speed photographs have been processed in MATLAB using the Control Point Selection Tool in 
the Imaging Processing Toolbox to remove any optical distortions. Before each experiment, a transparent sheet, with control points placed in a square grid pattern, was placed in the test section where the experimental specimen normally is located. The image of the grid with control points can then be corrected and then used to remove the optical distortions from the experimental photographs. The resolution of each image was $320 \times 128$ pixels and the time between subsequent frames was set to $8.38 \mu \mathrm{s}$. The corresponding measurement size of the specimen is $118.5 \mathrm{~mm} \times 47.4 \mathrm{~mm}$. During the $1 \mu \mathrm{s}$ minimum exposure time that the high-speed camera has, the shock wave in water will travel approximately $1.4 \mathrm{~mm}$, which can yield blurry images. To overcome this intrinsic limitation of the camera, a synchronized pulsed laser light source (SONY SLD1332V Laser diode combined with PicoLAS LDP-V 03-100UF3 Driver module) is used to provide intensive lighting within tens of nanosecond time scale. A typical pulse duration for the experiments is 40 ns, which means the exposure time is reduced to significantly reduce blur. During the experiments, when the event of interest takes place, a control signal is sent to a pulse/delay generator (BNC Model 575). The pulse generator receives the signal and outputs a sequence of TTL pulses to both the light source and the camera to synchronize the two.

\subsection{Background oriented schlieren}

The previous two types of visualization techniques are suitable for discovering flow features and performing basic flow related calculations such as the estimation of shock wave speed etc. However, it is preferable if the density or pressure change of the fluid is available for each experiment. As suggested by Meier (Meier, 2002), a technique called Background Oriented Schlieren 

are computed based on the wave speeds and density, $\rho$.

\begin{tabular}{cl|ccc} 
& & Polycarbonate & Aluminum & PMMA \\
\hline$\rho$ & {$\left[\mathrm{kg} / \mathrm{m}^{3}\right]$} & $1270^{\ddagger}$ & $2700 \ddagger$ & $1200^{\dagger}$ \\
$c_{L}$ & {$[\mathrm{~m} / \mathrm{s}]$} & $2100 \pm 110^{\ddagger}$ & $6370 \pm 120 \ddagger$ & $2800 \pm 55^{\dagger}$ \\
$c_{S}$ & {$[\mathrm{~m} / \mathrm{s}]$} & $950 \pm 50^{\ddagger}$ & $3150 \pm 60 \ddagger$ & $1500 \pm 30^{\dagger}$ \\
$E$ & {$[\mathrm{~Pa}]$} & $3.2 \times 10^{9}$ & $64 \times 10^{9}$ & $5.6 \times 10^{9}$ \\
$\nu$ & {$[-]$} & 0.37 & 0.31 & 0.34
\end{tabular}

Table 1: Material properties for the three core materials used in the experiments. ${ }^{\dagger}$ values measured using Olympus 38DL Plus, ${ }^{\ddagger}$ from Eliasson et al. (2010).

(BOS) can be incorporated with schlieren photography to yield quantitative density measurements in transparent flows.

\section{Experimental results}

Results have been obtained using the previous mentioned techniques; single-frame high-resolution schlieren photography, high-speed schlieren photography and high-speed BOS photography. Three different materials have been used, and their properties are summarized in Table 1. Transverse, $c_{S}$, and longitudinal, $c_{L}$, wave speeds are measured using an Olympus 38DL Plus Ultrasonic Thickness Gage, and Young's modulus and Poisson's ratio

\subsection{Polycarbonate specimen}

A polycarbonate core was used because it is transparent and therefore the wave propagation inside the core material can be visualized at the same time as the waves inside the water-filled region are investigated. A series of 


$$
\text { . }
$$

\begin{tabular}{ll|c|c|c|c|c|c|c|}
\multicolumn{2}{|c|}{ Frames } & $(\mathrm{b})-(\mathrm{c})$ & $(\mathrm{c})-(\mathrm{d})$ & $(\mathrm{d})-(\mathrm{e})$ & $(\mathrm{e})-(\mathrm{f})$ & $(\mathrm{f})-(\mathrm{g})$ & $(\mathrm{g})-(\mathrm{h})$ & $(\mathrm{h})-(\mathrm{i})$ \\
\hline PC: $u_{s}$ & {$[\mathrm{~m} / \mathrm{s}]$} & - & $1370^{*}$ & $1326^{*}$ & 1458 & 1547 & 1458 & - \\
\hline Al: $u_{s}$ & {$[\mathrm{~m} / \mathrm{s}]$} & 1724 & 1591 & 1591 & 1503 & 1503 & 1547 & 1503
\end{tabular}

Table 2: Speed of compression wave, $U_{s}$, between subsequent frames in Figure 2 for polycarbonate $(\mathrm{PC})$ with a projectile impact speed of $50.6 \pm 1.0 \mathrm{~m} / \mathrm{s}$, and Figure 4 for aluminum (Al) with a projectile impact speed of $50.7 \pm 1.0 \mathrm{~m} / \mathrm{s}$. All wave speeds have an uncertainty of $\pm 44 \mathrm{~m} / \mathrm{s}$, except values marked * with $\pm 88 \mathrm{~m} / \mathrm{s}$.

180

181

high-speed schlieren images with a projectile impact speed of $50.6 \pm 1.0 \mathrm{~m} / \mathrm{s}$ using the polycarbonate specimen is shown in Figure 2. Figure 2(a) shows the specimen before impact with the undisturbed water-filled region (marked $\mathrm{A}$ in the figure) and the blocker (marked B in the figure). The shock front is marked by white arrows. Time $t=0 \mu \mathrm{s}$ is when the shock wave enters the water region. The shock speed along the center line for frames (c)-(h) was measured using the distance between the shock front positions, and the timing between successive frames, and the result is presented in Table 2 . The error in shock speed, $\pm 44 \mathrm{~m} / \mathrm{s}$, is based on the uncertainty in shock location (1 pixel) divided by the time interval $(8.38 \mu \mathrm{s})$, and the Mach number error was calculated using error propagation rule for $M_{s}=u_{s} / c_{\text {water }}$ where $c_{\text {water }}=1491 \pm 2.8 \mathrm{~m} / \mathrm{s}$. The first two frames indicate a wave speed less than

Mach 1, which is likely caused by the strong interaction of the pressure and shear waves in the windows. There is a larger error attributed to determining the shock location ( 2 pixels) for these images than the rest since the region where the shock wave is located is smeared out and hard to precisely locate.

In Figure 2(g) the angle between the interface of the convergent section and the shear waves in the polycarbonate core was measured to be $34^{\circ} \pm 1^{\circ}$ 
on the upper edge and lower edge. From such an angle, one can estimate the shock wave speed in water by assuming that the shear wave remains constant in the surrounding solid (Eliasson et al., 2010). In later frames, Figure $2(\mathrm{~h})$, this angle has decreased to $29^{\circ} \pm 1^{\circ}$. This decrease is due to the curvature change of the fluid-solid interface, and if the horizontal component of the shear wave is computed it is clear that the shock speed in water remains constant. Therefore, a transparent sample can be used to further help understand the instantaneous wave propagation inside the water-filled region. In the last frame, $2(\mathrm{j})$, a cylindrical wave (marked by a white arrow) has started to propagate into the core material as a result of the shock focusing event in the water-filled region.

A single-shot schlieren experiment is presented in Figure 3. This image is taken at a late time when there are extensive cavitation zones both inside the convergent water-filled cavity, and in the thin liquid films between the windows and the core material. The dark zone behind the shock wave is due to extensive disturbances, and all light is diverted from the schlieren optical path. The white arrows point at the curved shear wave in the polycarbonate core, and their symmetry is a sign of a planar impact. A curved shear wave is formed due to the nonlinear geometry of the interface between the fluid and the solid.

\subsection{Aluminum specimen}

An aluminum core material was chosen for its ability to provide more realistic wave interactions as seen in marine applications. A series of highspeed schlieren images with a projectile impact speed of $50.7 \pm 1.0 \mathrm{~m} / \mathrm{s}$ using an aluminum specimen is shown in Figure 4. Figure 4(a) shows the specimen 
before impact with the undisturbed water-filled region (marked $\mathrm{A}$ in the figure) and the blocker (marked $\mathrm{B}$ in the figure). The dark region in the water-filled region of Figure 4(a)-(d) is caused by the interference of light from the laser diode, and it can be removed by using a shadowgraph technique instead of a schlieren technique. The shock speed along the center line for frames (b)-(i) was measured similarly as for the polycarbonate specimen, and the results are presented in Table 2.

Figure 5 shows a single-shot schlieren experiment with aluminum as surrounding core material. The projectile impact speed of this test is at $79 \pm 1 \mathrm{~m} / \mathrm{s}$. The arrows point at (1) the shock wave in water, (2) the oblique precursor wave from the shear wave in the aluminum core material, and (3) the pressure wave in the polycarbonate windows. Cavitation bubbles occur between the precursor wave and the main shock front due to the interaction between the faster traveling stress waves inside the surrounding structure.

\subsection{PMMA specimen}

A PMMA core specimen, with a reduced thickness to one quarter of the previous cores, was used together with a background oriented schlieren method to measure density and pressure along the centerline of the waterfilled region. In this particular setup, any pressure sensor that is physically attached to the core material is subjected to the risk of getting hit and damaged during the experiment, and this was determined unfeasible. Therefore, a non-intrusive visual technique, BOS, was chosen to gain knowledge of the density and pressure evolution in the convergent water-filled section, see details in Wang et al. (2013). The thickness was reduced to realize an improved BOS result because the pressure from BOS is calculated under the 
assumption that the experimental domain is two-dimensional and thus threedimensional effects were reduced by reducing the core thickness. Other dimensions of the PMMA samples stay the same as previous experiments. The projectile impact speed during the test is $50.0 \pm 1.0 \mathrm{~m} / \mathrm{s}$. Two-dimensional pressure distribution at time $54.5 \mu \mathrm{s}$ is reconstructed by feeding the displacement information into a solver by Wang et al. (2013). Two extremes of boundary conditions were used to determine the maximum (16 MPa) and minimum (10 MPa) pressure peaks. The resulting pressure profile along the center axis of the logarithmic spiral geometry for the boundary conditions yielding the lower peak pressure is plotted in Figure 6. There is a $4 \mathrm{~mm}$ spatial span of the pressure rise as seen in Figure 6, which is due to the finite window size of the image analysis. Behind the pressure peak, the pressure drops to 3.9 MPa, which corresponds to a long trailing expansion wave. Also, compared to the previously presented experimental results by Wang et al. (2013), using a similar projectile impact speed, the calculated peak pressure for the logarithmic spiral presented here is about three times higher than for their wedge-shaped cavity made of polycarbonate. 

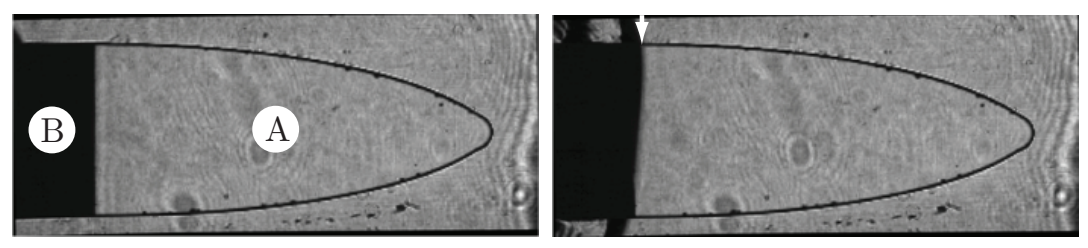

(a) Before test

(b) $t=0 \mu \mathrm{s}$
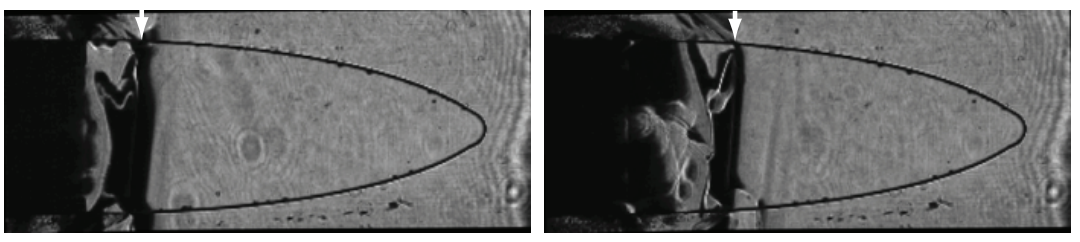

(c) $t=8.38 \mu \mathrm{s}$

(d) $t=16.76 \mu \mathrm{s}$
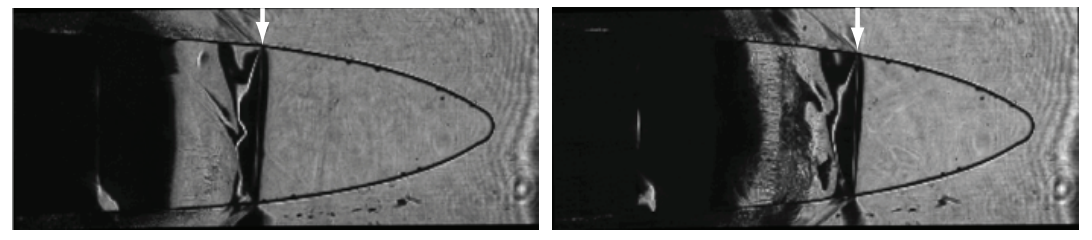

(e) $t=25.14 \mu \mathrm{s}$

(f) $t=33.52 \mu \mathrm{s}$
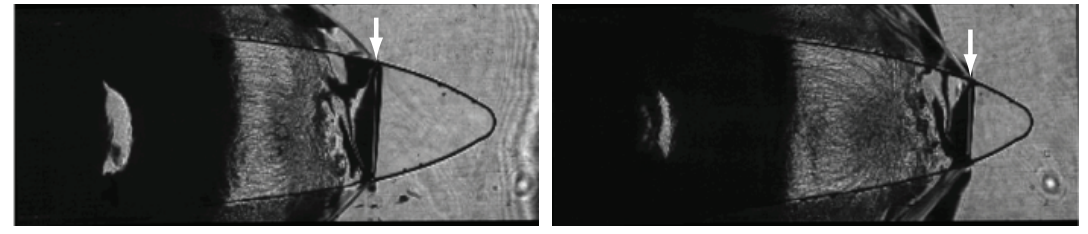

(g) $t=41.90 \mu \mathrm{s}$

(h) $t=50.28 \mu \mathrm{s}$
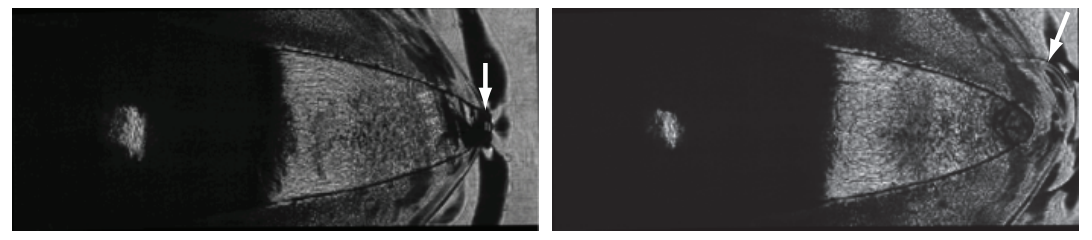

(i) $t=58.66 \mu \mathrm{s}$

(j) $t=67.04 \mu \mathrm{s}$

Figure 2: A series of high-speed schlieren images using a polycarbonate specimen. Projectile impact speed of $50.6 \pm 1.0 \mathrm{~m} / \mathrm{s}$. The white arrow shows the location of the shock front in (b)-(i), and the pressure wave in the core material in (j). 


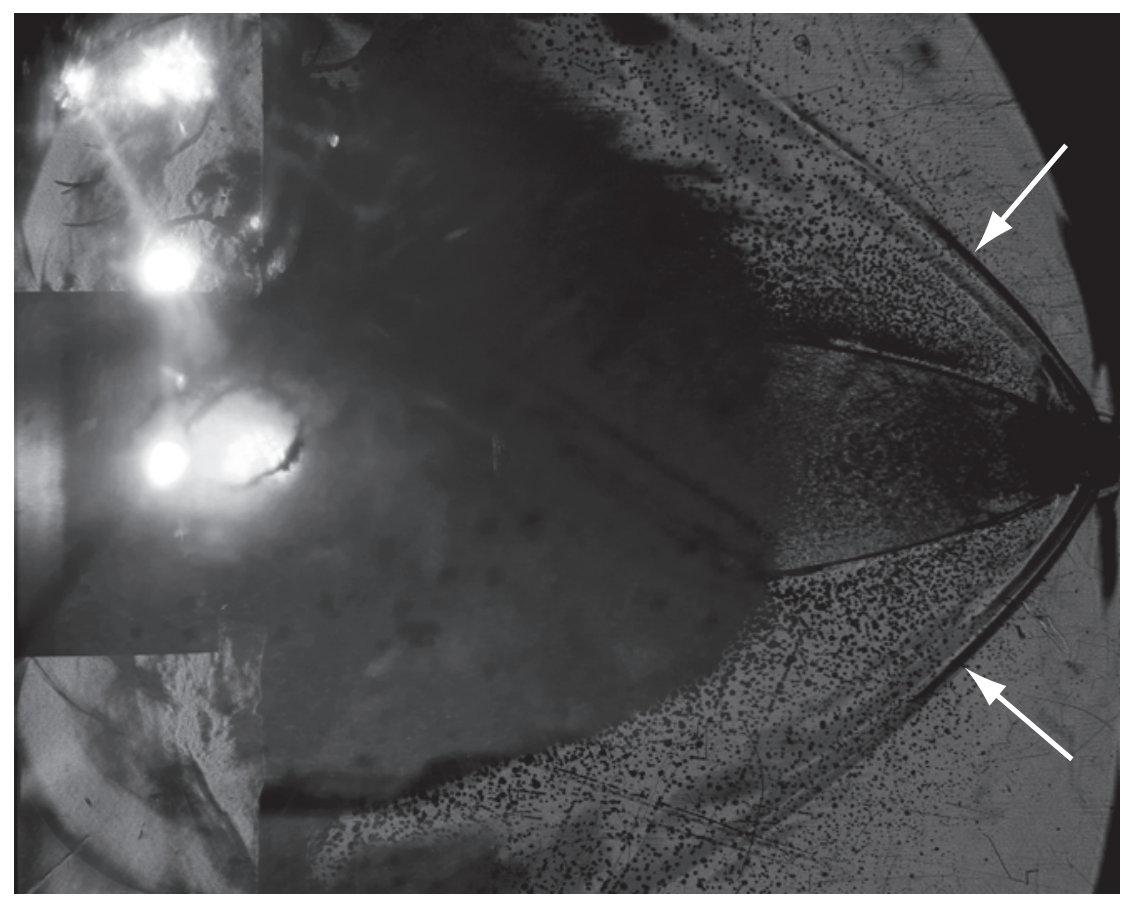

Figure 3: A single-shot schlieren experiment using a polycarbonate specimen visualized at $t=111.4 \mu \mathrm{s}$ after the shock wave enters the water-filled region. Image shows extensive cavitation zones, not just in the water-filled cavity but also in the thin liquid layers between the windows and the core material. White arrows point at shear waves inside the polycarbonate core material. 


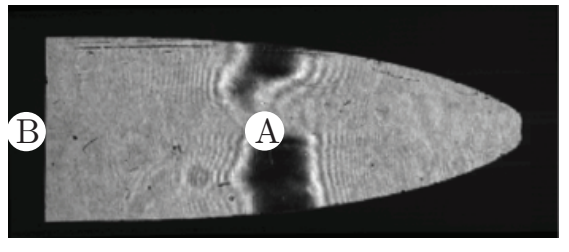

(a) Before test

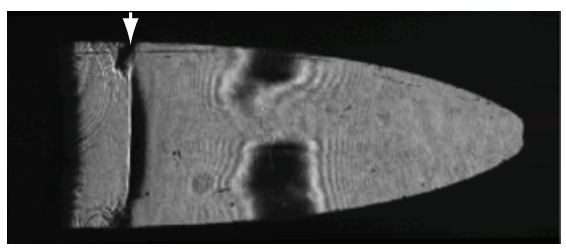

(c) $\Delta t=8.38 \mu \mathrm{s}$

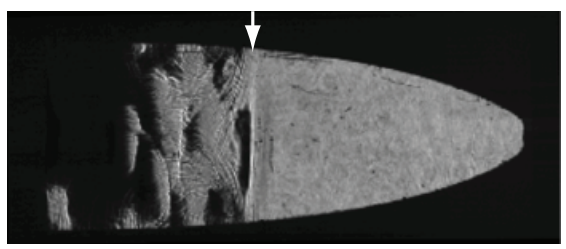

(e) $\Delta t=25.15 \mu \mathrm{s}$

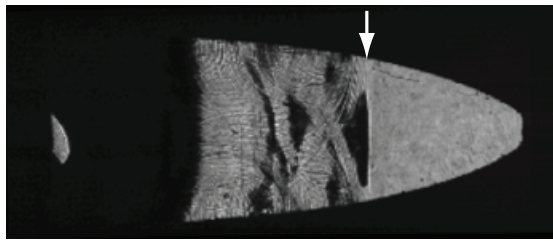

(g) $\Delta t=41.91 \mu \mathrm{s}$

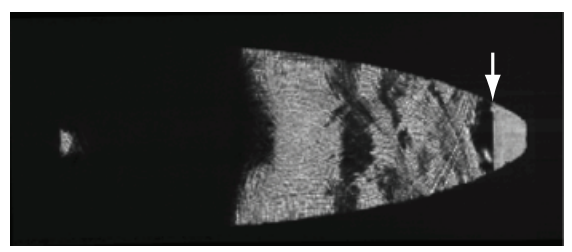

(i) $\Delta t=58.68 \mu \mathrm{s}$

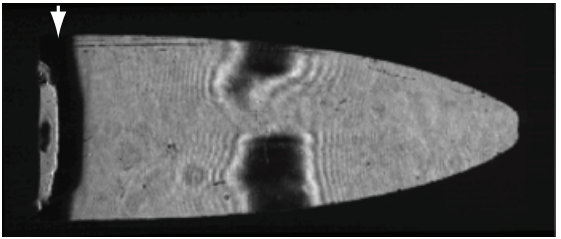

(b) $\Delta t=0 \mu \mathrm{s}$

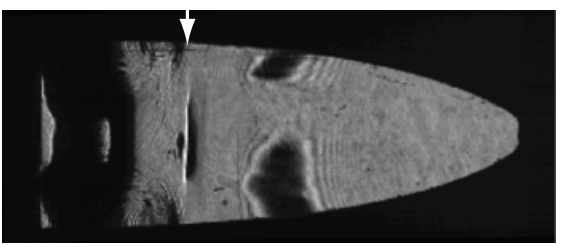

(d) $\Delta t=16.76 \mu \mathrm{s}$

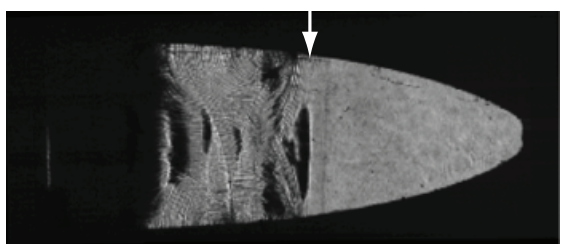

(f) $\Delta t=33.53 \mu \mathrm{s}$

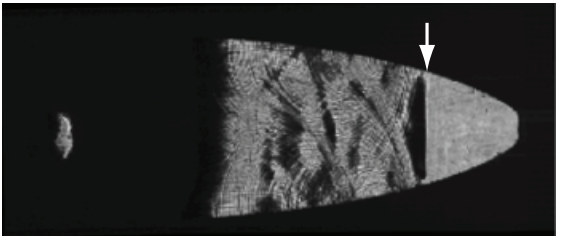

(h) $\Delta t=50.29 \mu \mathrm{s}$

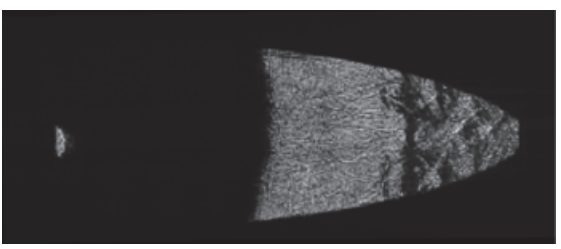

(j) $\Delta t=67.06 \mu \mathrm{s}$

Figure 4: A series of high-speed schlieren images using an aluminum specimen. Projectile impact speed of $50.7 \pm 1.0 \mathrm{~m} / \mathrm{s}$. The white arrow shows the location of the shock front in (b)-(i). 


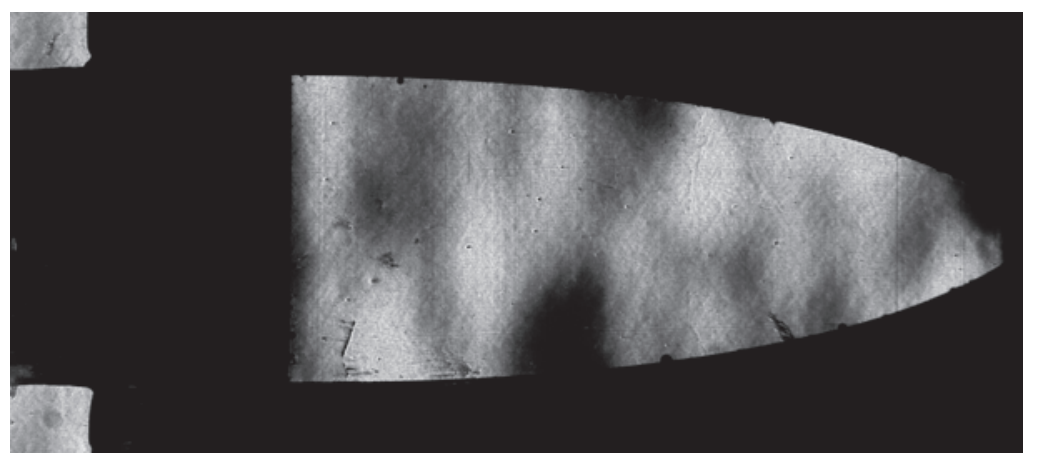

(a) Before test

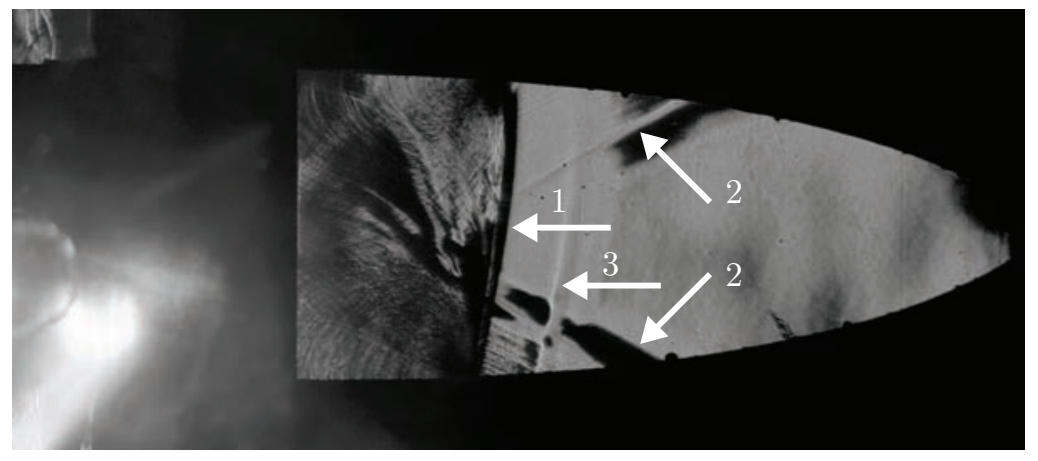

(b) $t=39.4 \mu \mathrm{s}$

Figure 5: A single-shot schlieren experiments using an aluminum specimen with projectile impact speed $79 \pm 1 \mathrm{~m} / \mathrm{s}$. Arrows point at (1) shock wave in water; (2) oblique precursor waves from the shear wave in the aluminum core material, and (3) pressure wave in the polycarbonate windows. 


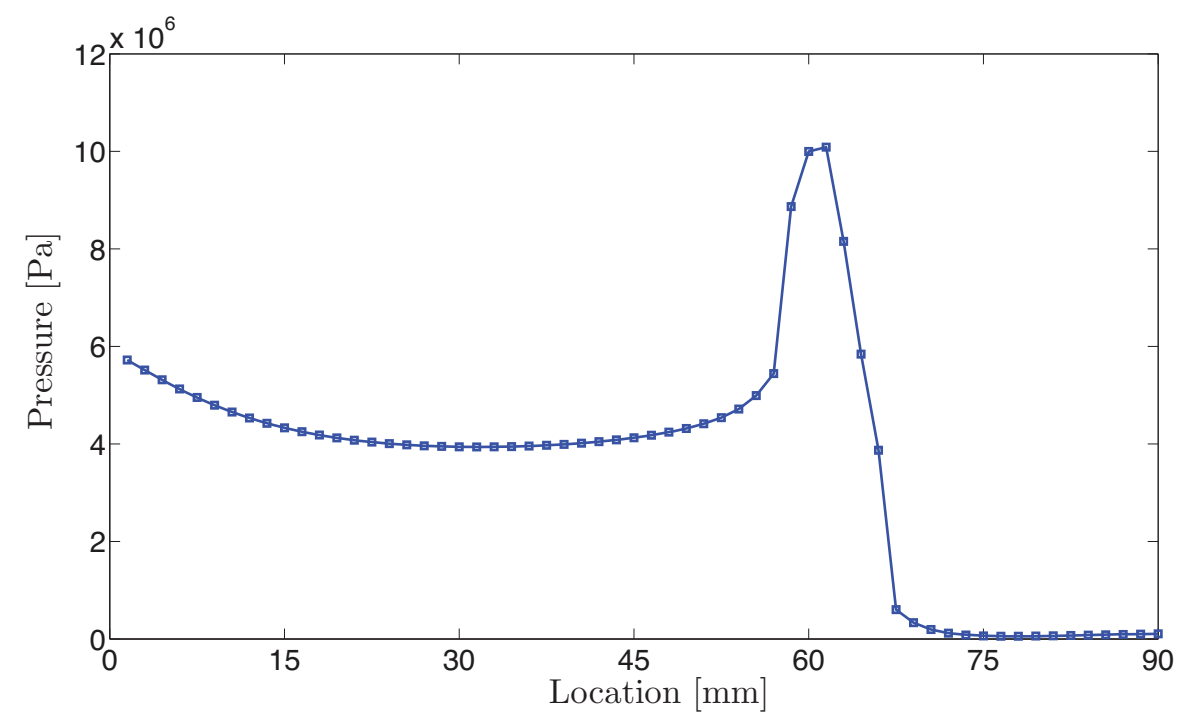

Figure 6: The reconstructed pressure measurement along the center line. 


\section{Numerical method and experiments}

In this section, results from three different types of simulations of converging shocks in water are presented; (4.1) a two-dimensional rigid confinement, (4.2) a two-dimensional multi-physics simulation that accounts for the fluidstructure interaction, and (4.3) an axisymmetric rigid confinement.

The Overture package, a finite difference code for partial differential equations, is utilized to perform all the simulations (Chesshire and Henshaw, 1990). The inviscid and adiabatic flows under consideration are governed by the Euler equations of gas dynamics, i.e. conservation of mass, momentum and energy,

$$
\frac{\partial \rho}{\partial t}+\nabla \cdot(\rho \mathbf{u})=0
$$

$$
\frac{\partial(\rho \mathbf{u})}{\partial t}+\nabla \cdot(\rho \mathbf{u u})+\nabla p=0
$$

$$
\frac{\partial(\rho E)}{\partial t}+\nabla \cdot(\mathbf{u}(\rho E+p))=0
$$

$$
E=e+\frac{1}{2}\left(u^{2}+v^{2}+w^{2}\right)
$$

where $\rho, \mathbf{u}, p$ are the density, velocity vector and pressure. In Equation (4), $E$ and $e$ are the total energy and the internal energy per unit mass, and $u, v, w$ are the velocity components in three directions. A stiffened equation of state (sometimes referred to as Tammann-Tait EOS, Ridah (1988)) is used to close the system of equations. The stiffened EOS is given by

$$
e=\frac{p+\gamma_{\infty} p_{\infty}}{\rho\left(\gamma_{\infty}-1\right)}
$$

The variable $e$ is the internal energy, $p$ is the pressure, $\rho$ is the density; and the constants $\gamma_{\infty}=7.415, p_{\infty}=2.962 \times 10^{8} \mathrm{~Pa}$ are chosen to match the speed 
of sound of water in previous laboratory experiments reported by Eliasson (Eliasson et al., 2010). This EOS has also been used in previous works related to shock waves in water, such as underwater explosions (Cocchi et al., 1996) and the collapse of shock-induced bubbles in water (Johnsen and Colonius, 2008).

The Rankine-Hugoniot jump conditions using the stiffened EOS are represented by the following equations

$$
\begin{gathered}
u_{2}=a_{1} \frac{2\left(M_{s}^{2}-1\right)}{\left(\gamma_{\infty}+1\right) M_{s}}, \\
\rho_{2}=\rho_{1} \frac{\left(\gamma_{\infty}+1\right) M_{s}^{2}}{\left(\gamma_{\infty}-1\right) M_{s}^{2}+2}, \\
p_{2}=\rho_{1} a_{1}^{2} \frac{2\left(M_{s}^{2}-1\right)}{\gamma_{\infty}+1}+p_{1} .
\end{gathered}
$$

Here, $M_{s}$, is the shock Mach number, $u$, is the particle velocity and $a_{1}$ is the speed of sound. Quantities with a subscript 1 denote variables in the undisturbed region ahead of the shock wave and quantities with a subscript 2 denotes variables in the region behind the shock.

The conservation equations are solved numerically using a shock-capturing second-order Godunov method (Henshaw and Schwendeman, 2003, 2006). Composite grids are generated by overlapping structured grids (Henshaw and Schwendeman, 2006). The main reason for using composite grids is to save memory because the main part of all grids are rectangular Cartesian grids which are more efficient in terms of memory usage than curvilinear grids (Chesshire and Henshaw, 1990).

A measure of interest is the peak pressure at the tip of the logarithmic spiral, i.e. the focal region. This peak pressure is an indicator of the destructive potential of the converging shock wave to the surrounding structure. 
Transient high pressures will induce strong tensile and shear stresses inside the surrounding solid. The stress field can in turn cause initiation and growth of any defects already present in the structure, which eventually can lead to crack formation (Xi and Zhong, 2001) with devastating results.

A grid sensitivity study using Richardson Extrapolation theory has been performed on the two-dimensional rigid boundary case (details explained in section 4.1). Four grid sizes were used, each one half the size of the previous: $0.25 \mathrm{~mm}, 0.125 \mathrm{~mm}, 0.0625 \mathrm{~mm}$, and $0.03125 \mathrm{~mm}$. Pressure history was recorded at location $(x, y)=(-4,0)$, which is close to the tip, located at $(0.0)$, of the logarithmic spiral. The non-dimensionalized peak pressure (multiply with 2196 to get result in $\mathrm{MPa}$ ) is plotted against $\Delta x^{2}$ in Figure 7 , and a nearly linear relation is obtained, which is in agreement with the result a second order Godunov scheme theoretically gives. In addition, an Adaptive Mesh Refinement (AMR) algorithm in Overture is also utilized to capture the fine structures of the shock focusing process. The AMR grids are two levels with a refinement factor of four each, which results in an eight times smaller grid size near shocks, contact surfaces and other regions with sharp derivatives. Therefore, a grid size of $0.1 \mathrm{~mm}$ was used to balance the cost of the simulation and the accuracy of the result.

All simulations presented below are first run with an incident shock Mach number of $M_{s}=1.1$ because the logarithmic spiral is designed to most efficiently converge the shock for this specific Mach number. Then, simulations are repeated with a lower shock Mach number of $M_{s}=1.03$, which is closer to the one realized in the experiments. 


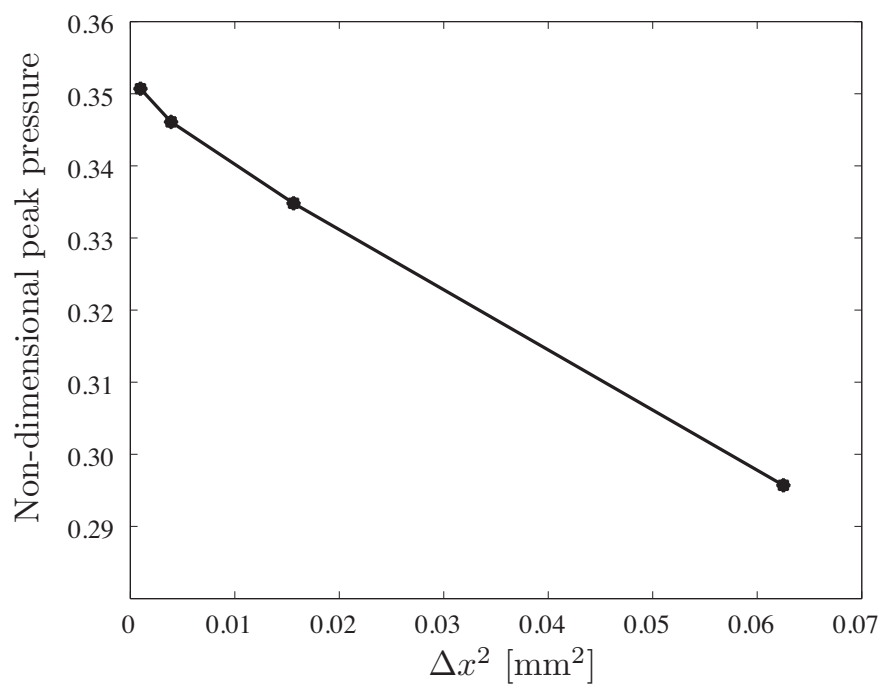

Figure 7: Grid sensitivity study by investigating peak pressures for different initial grid sizes.

\subsection{Two-dimensional rigid boundary simulation}

This type of simulation, in which only the liquid part of the problem is considered without any influence from the surrounding solid structure, is informative in two aspects. First, it enables the possibility to investigate how the geometry influences the shock focusing event. Second, it should give the ideal maximum conditions at the focal point for two-dimensional simulations since there is no energy lost to the surrounding structure.

To perform the simulation, a composite grid made of three different grids overlapping each other is prepared, Figure 8(a). A rectangular Cartesian grid is first generated as a background grid. All other grid components cut or replace part of the background grid. The reflector grid giving the outer boundary of the logarithmic spiral is obtained by marching from a spline whose points are given by the equation for a logarithmic spiral. The reflector 
325 grid.

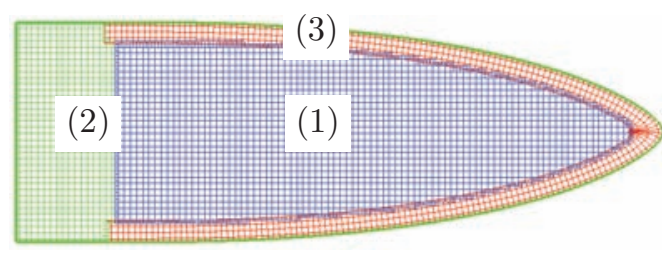

(a)

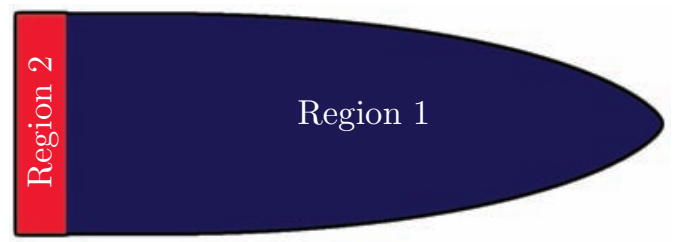

(b)

Figure 8: (a) An overview of the composite grid used for two-dimensional rigid boundary simulations with (1) Cartesian background grid; (2) inlet grid; (3) logarithmic spiral boundary grid. (b) Initial conditions in region 1 ahead of the shock wave, and region 2 behind the shock wave summarized in Table 3 .

Step functions of pressure, $p$, velocity, $u$, and density, $\rho$, are used as initial conditions to represent an initially planar incident shock wave. The conditions in the undisturbed region ahead of the shock wave are given by room temperature $\left(21^{\circ} \mathrm{C}\right)$, atmospheric pressure $(101,325 \mathrm{kPa})$ and zero velocity. The conditions behind the shock wave are computed using shock jump conditions, Equations (6)-(8), based on the chosen shock Mach number, $M_{s}=1.1$, and the conditions ahead of the shock. The initial conditions are summarized in Table 3, and the shock location at time $t=0$ is shown in Figure 8(b). At the left boundary of the inlet grid, a constant inflow condition (given by region 2 in Table 3) is maintained to sustain the shock wave strength. The boundary condition at the wall profile is set to be a slip-wall condition, which keeps the normal velocity of the fluid zero at the wall boundary. As a consequence of this slip-wall boundary condition, the shock front is always perpendicular to the wall, as shown in Figure 9. 


\begin{tabular}{l|c|c} 
& Region 1 & Region 2 \\
\hline$p[\mathrm{MPa}]$ & 0.1 & 109.8 \\
$u[\mathrm{~m} / \mathrm{s}]$ & 0 & 67 \\
$\rho\left[\mathrm{kg} / \mathrm{m}^{3}\right]$ & 1000 & 1043
\end{tabular}

Table 3: Initial conditions in region 1 ahead of the shock wave, and region 2 behind the shock wave.

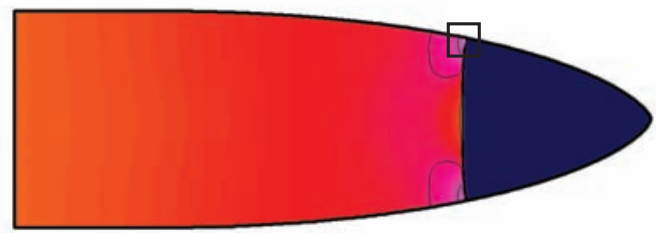

(a)

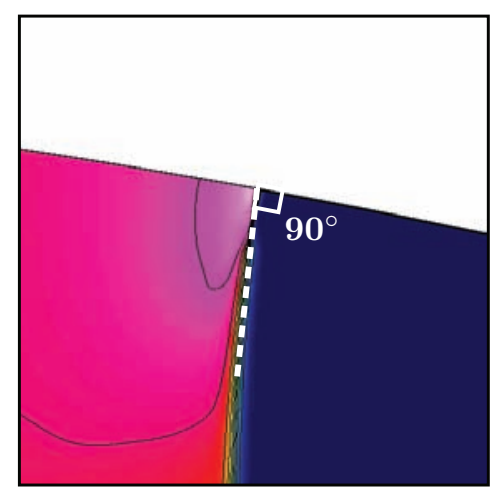

(b)

Figure 9: (a) A snapshot of pressure distribution at time $40.5 \mu \mathrm{s}$. (b) Zoom in of the shock front from (a) showing that the shock front is normal to the wall as a consequence of applying slip-wall condition at the boundary.

Four snapshots at various time instances are shown in Figure 10. As the shock wave propagates, the Mach number of the shock front at the boundary increases due to the slope of the boundary. The observed peak pressure at the tip is $4.3 \mathrm{GPa}$. It is 39.2 times the initial pressure, $p_{2}$, behind the shock wave. An additional simulation with initial shock Mach number $M_{s}=1.03$ was conducted to match the experimental conditions, and the maximum pressure in this case is $319.8 \mathrm{MPa}$ at the focal point. To compare with the 


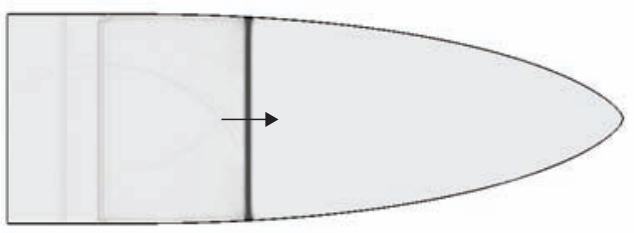

(a) $t=20.2 \mu \mathrm{s}, M_{s}=1.13, p=118 \mathrm{MPa}$

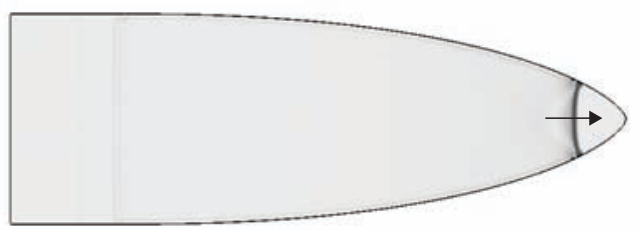

(c) $t=54.0 \mu \mathrm{s}, M_{s}=1.32, p=228 \mathrm{MPa}$

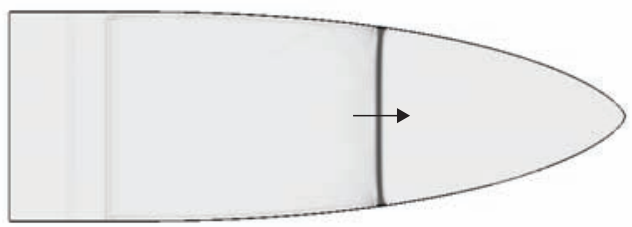

(b) $t=33.7 \mu \mathrm{s}, M_{s}=1.26, p=136 \mathrm{MPa}$

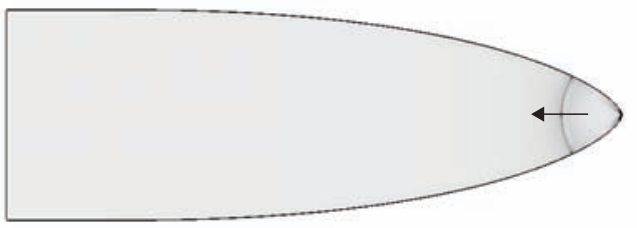

(d) $t=63.4 \mu \mathrm{s}, M_{s}=1.60, p=2.5 \mathrm{GPa}$

Figure 10: Schlieren plots at different time instants. The shock Mach number and pressure right behind the shock wave increases as the shock converges. The arrow points in the direction of the wave propagation.

BOS results, the maximum pressure was also measured $30 \mathrm{~mm}$ away from the tip. In the case where $M_{s}=1.1$, the maximum pressure is $124.5 \mathrm{MPa}$, and for $M_{s}=1.03$ it is $36 \mathrm{MPa}$.

\subsection{Two dimensional fluid-structure interaction simulation}

To better understand the loads and the coupling on the surrounding structure, a two-dimensional fluid-structure interaction simulation is performed. This is a coupled problem because the shock generated in the water results in both longitudinal and transversal waves in the surrounding material. In turn, the longitudinal and transversal waves in the solid interact with the water.

The governing equations for the fluid part is the same as in the first case with a rigid confinement. Linear elasticity is assumed in the solid part and 
the dynamics of the displacement is given by

$$
\begin{gathered}
\rho \frac{\partial^{2} u_{i}}{\partial t^{2}}=\frac{\partial \sigma_{i j}}{\partial x_{j}}+\rho f_{i}, \\
\sigma_{i j}=\lambda\left(\epsilon_{k k}\right) \delta_{i j}+2 \mu \epsilon_{i j} .
\end{gathered}
$$

In Equation (9), $u_{i}, \sigma_{i j}$ and $f_{i}$ are the displacement function of material points in direction $i$, the Cauchy stress tensor, and the body force in direction $i$. And in Equation (10), $\epsilon_{i j}$ is the strain tensor, while $\lambda$ and $\mu$ are the two Lamé constants. The material properties of the aluminum core are shown in Table 1. The grid size is set to $\Delta x=1 \mathrm{~mm}$.

The composite grid for this type of simulation consists four parts including a Cartesian grid for the fluid domain, a Cartesian grid for the solid domain, and two curvilinear grids for communication between the fluid and the solid, Figure 11. The boundary condition between the two curvilinear boundary grids is a traction boundary condition, which means that the normal stress at the fluid-solid interface is matched. For initial conditions, a shock jump is set in the fluid domain as in the previous case with the rigid boundary. The solid part is left undisturbed. As the shock wave starts to propagate into the logarithmic spiral duct, it will deform the interface between the fluid and the solid region. Because the fluid will be moving together with the deforming boundary to maintain contact, the overall velocity of the fluid behind the shock wave will be moving toward the undisturbed boundary. As a consequence, the shock front has to be at an angle less than $90^{\circ}$ to the boundary to balance this flow condition (Arienti et al., 2003), Figure 12. Energy carried by the shock wave is attenuated by the transmission of energy into solid, 


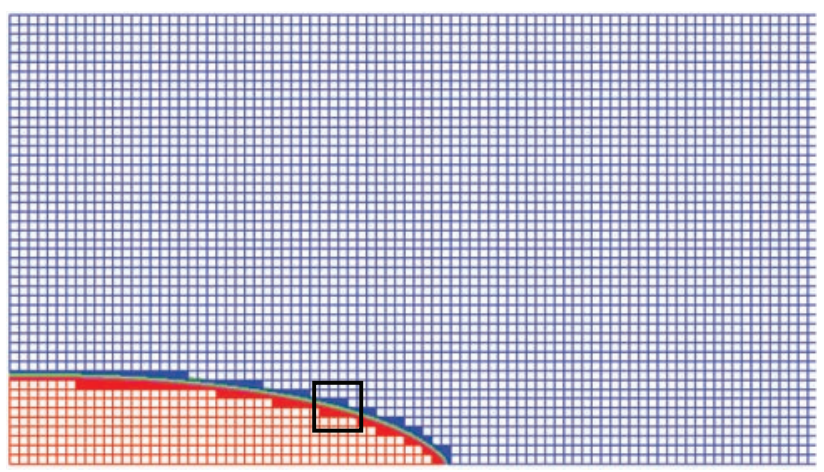

(a)

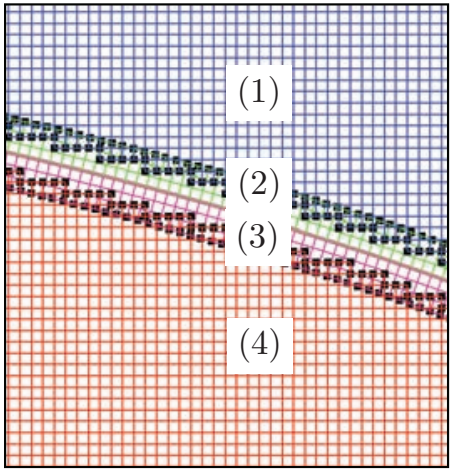

(b)

Figure 11: (a) An overview of the composite grid for the FSI simulation. The solid colored blocks are finer grids at the interface. (b) A blowup of the marked region of the interface from (a). (1) solid domain; (2) boundary grid for the solid domain; (3) boundary grid for the fluid domain; (4) fluid domain. Interpolation points are marked as black dots. The boundary condition between grid (1) and grid (2) is given by a traction boundary condition.

Figure 12. This results in a lower peak pressure of $900 \mathrm{MPa}$ at the focal region as compared to the previous result from the rigid boundary simulation with a peak pressure of $4.3 \mathrm{GPa}$, because energy is transmitted from the liquid region to the surrounding solid. When the shock Mach number is lowered to $M_{s}=1.03$ the peak pressure is reduced to $320 \mathrm{MPa}$. Detailed plots of pressure along the interface for the incoming and reflected shock waves are given in Figure 13. The pressure in front of the incoming shock wave is lower than zero, due to the interaction with the pre-cursor waves, which indicates the possibility of cavitation formation. This feature is not observed in the two-dimensional rigid boundary simulation, which completely lacks pre-cursor waves.

A comparison with the results from this type of simulation and the ex- 


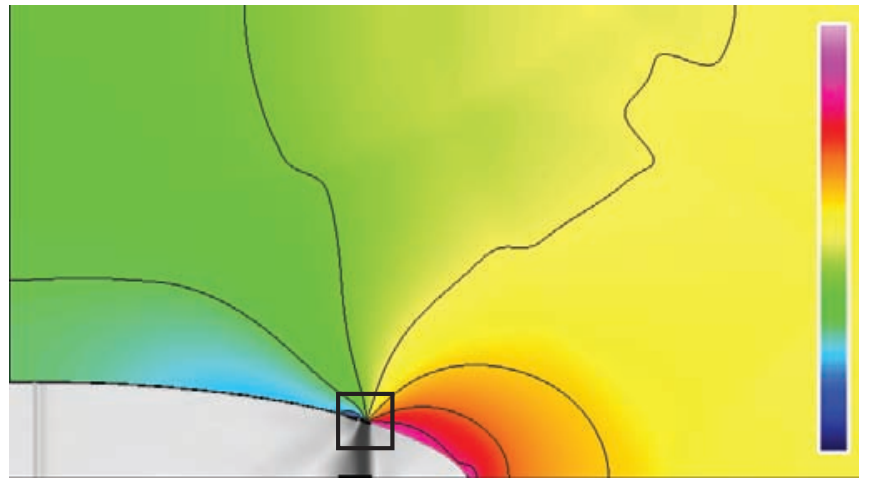

(a)

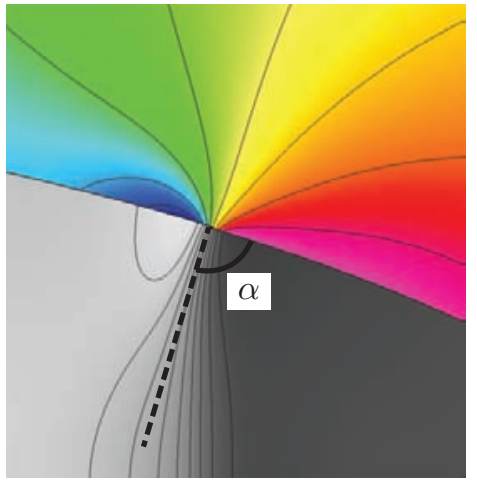

(b)

Figure 12: (a) A snapshot of the FSI simulation at time $t=43.5 \mu \mathrm{s}$. Schlieren is plotted inside the water-filled region and the divergence of the displacement field is plotted in the solid domain. A color bar is inserted to show which colors correspond to high values (pink) and low values (blue). (b) Zoom in of shock front at the interface from (a) showing that the shock front is not perpendicular to the interface. Instead, the shock angle $\alpha$ is $88.2^{\circ}$ for this particular time instance.

periments performed on the aluminum specimen show similar trends for the shock wave speed during the focusing phase. The simulated shock wave speeds are calculated at the same spatial locations as the high-speed visualization results shown in Figure 4, see Table 4. The general trends observed for the shock wave front are a deceleration of shock wave speed and also a dispersion in space, which is in agreement with the experimental observation.

The present results take the fluid-solid interaction into account but not three-dimensional effects. In order to understand how three-dimensional effects influence the peak pressure at the focal region an axi-symmetric simulation has been performed because in reality this would indicate the maximum pressure possible in this type of logarithmic spiral configuration. 


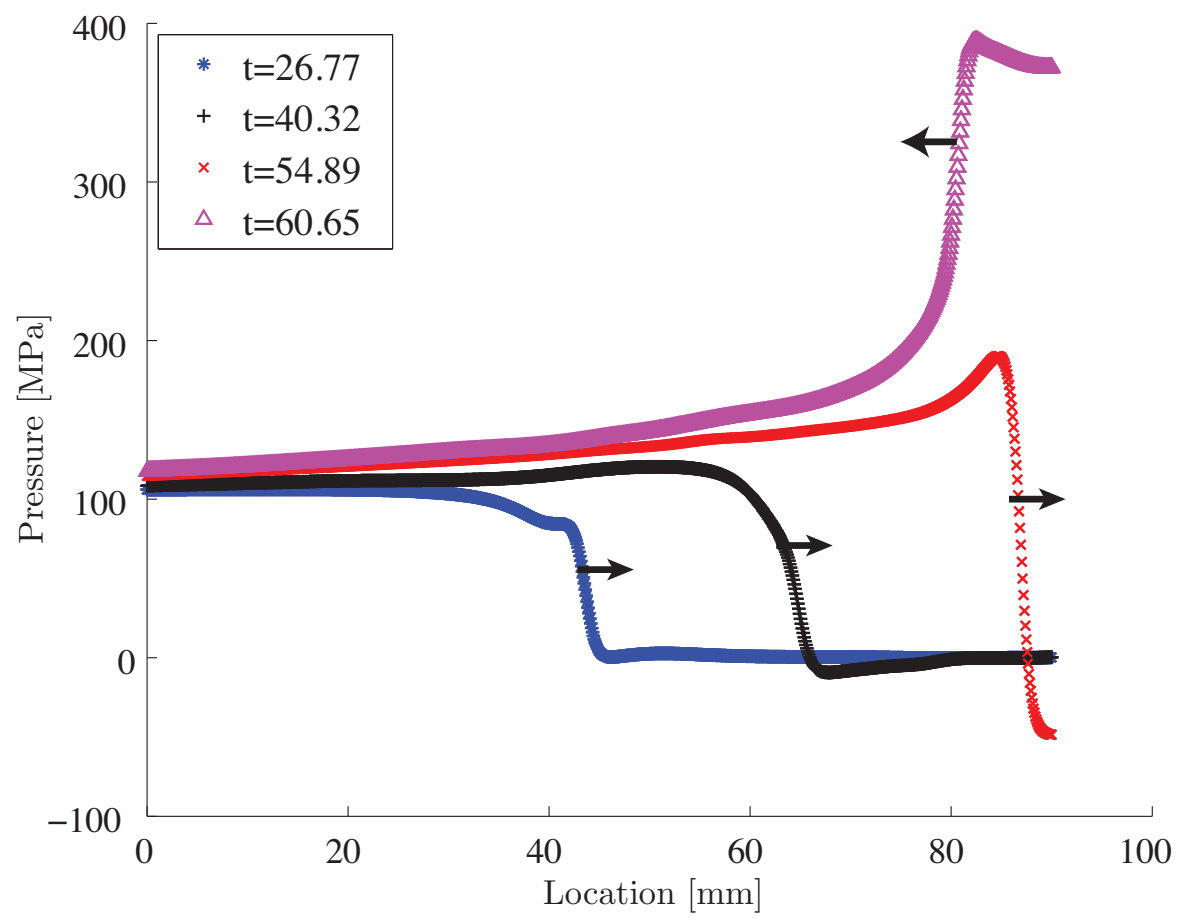

Figure 13: Pressure plots along the interface at time instants $t=26.77,40.32,54.89$, $60.65 \mu \mathrm{s}$. The peak pressure increases as the shock propagates towards the focal region, and again after reflection.

\subsection{Axisymmetric simulation}

A cylindrical symmetric or so-called axisymmetric coordinate system is used to perform the simulation. The axis of rotational symmetry is along the line where the characteristic length $L$ is defined, Figure 1. Accordingly, the governing equations are also expressed in axisymmetric coordinates. Because the solution does not depend on the rotational angle (there is no swirl in the solution, Henshaw (2003)), the equations can be discretized and solved on a two-dimensional mesh. The center line of the logarithmic spiral is set as axis of symmetry. Slip-wall boundary conditions are again applied to the 


\begin{tabular}{c|c|c|c} 
& Experiment [m/s] & Simulation [m/s] & Difference [\%] \\
\hline (c)-(d) & 1591 & 1642 & 3.1 \\
(d)-(e) & 1591 & 1640 & 3.0 \\
(e)-(f) & 1503 & 1639 & 8.3 \\
(f)-(g) & 1503 & 1515 & 0.8 \\
(g)-(h) & 1547 & 1480 & 4.5 \\
(h)-(i) & 1503 & 1515 & 0.8
\end{tabular}

Table 4: Shock wave speed comparison between the aluminum specimen and the twodimensional fluid-structure interaction simulation.

wall boundary, and constant inflow conditions are assigned to the in-flow boundary.

The resulting peak pressure at the focal region in the axisymmetric simulation is $34.2 \mathrm{GPa}$ for $M_{s}=1.1$ and $6.2 \mathrm{GPa}$ for $M_{s}=1.03$, which is higher than that of the both previously described simulations. The main reason for this high peak pressure is that a larger amount of energy carried by the shock wave is brought to the focal region as compared with the two previous cases. From the discussion above, much higher peak pressure can be achieved by using a three-dimensional logarithmic spiral shape, which suggests the significance of including the three-dimensional effects.

\section{Discussion}

Three materials were used for these experiments, and the clear plastics were chosen to visualize the waves inside the water-filled region and simultaneously visualize the longitudinal and shear waves inside the core material. A benefit from visualizing the transverse waves is that they provide an es- 

to two-dimensional.

\begin{tabular}{l|c|c|c} 
Simulation & $M_{s}$ & $p_{\max }^{30 \mathrm{~mm}}$ & $p_{\max }^{\text {tip }}$ \\
\hline 2D, Ridgid & 1.03 & $36 \mathrm{MPa}$ & $319.8 \mathrm{MPa}$ \\
2D, Ridgid & 1.1 & $124.5 \mathrm{MPa}$ & $4.3 \mathrm{GPa}$ \\
Axi-symmetric, Rigid & 1.03 & $42.2 \mathrm{MPa}$ & $6.2 \mathrm{GPa}$ \\
Axi-symmetric, Rigid & 1.1 & $146.3 \mathrm{MPa}$ & $34.2 \mathrm{GPa}$ \\
2D, FSI, Aluminum & 1.03 & $34.3 \mathrm{MPa}$ & $149.7 \mathrm{MPa}$ \\
2D, FSI, Aluminum & 1.1 & $115 \mathrm{MPa}$ & $900 \mathrm{MPa}$ \\
\hline \hline PMMA, BOS & - & $13 \pm 3 \mathrm{MPa}$ & -
\end{tabular}

Table 5: Maximum pressure summary for simulations and BOS experiment. Simulation results are obtained in the three different types of simulations for two shock Mach numbers $\left(M_{s}=1.1\right.$ and $\left.M_{s}=1.03\right)$, and at two locations: at the tip of the logarithmic spiral, $p_{\max }^{\text {tip }}$, and $30 \mathrm{~mm}$ before the tip, $p_{\max }^{30 \mathrm{~mm}}$.

434 The aluminum core was used since it closer replicates the conditions for marine applications where shock focusing can occur. The maximum pressures obtained in the simulations and the BOS experiment are summarized in Table 5. Clearly, if a rigid surrounding is assumed, the maximum pressure will be higher than if the energy can be transmitted to a surrounding structure. The results of the axi-symmetric simulation also indicates that three-dimensional effects are important, however, the experiments are performed in planar geometries where the shock focusing in assumed to be close

The maximum pressure for the BOS measurement is about three times lower than the results from the rigid two-dimensional simulation with $M_{s}=$ 1.03, and there are several reasons for this result. First, the fluid-structure

timate of the instantaneous shock Mach number in the water-filled region. 
interaction has not been taken into account, which will reduce the simulated pressure further. Second, in the BOS evaluation, a two-dimensional assumption is used, which is likely not accurate since there are waves propagating out-of-plane, towards and through the windows and back. Third, while evaluating the BOS result, a density change of $4 \mathrm{~kg} / \mathrm{m}^{3}$ was measured and then a pressure increase was inferred from an equation of state taken from open literature Wagner and Pruß (1995). It is possible that if a more elaborate EOS was used, the calculated pressure would change.

The FSI simulation requires longer computational time, but provides more accurate results. The cavitation bubbles seen in the high-speed photographs in front of the incident shock wave for the aluminum experiments can be correlated to the lower pressure ahead of the front of the shock wave visualized in the FSI pressure plots in Figure 13. This result is also in agreement with the experimental results from Eliasson et al. (Eliasson et al., 2010), which showed clear evidence of precursor waves in the water ahead of the moving shock wave when a convergent aluminum confinement was used. The precursor waves occurred due to faster longitudinal and transversal wave propagation in the surrounding solid compared to the shock wave speed in water. Also, if the surrounding structure is smaller or thinner such that waves reflecting from its outer boundary come back sooner then the coupling with the liquid-filled region will be stronger. The surrounding structure can also deform if it is thinner or made of a softer material, and energy will then dissipate quicker and the shock will not generate as high pressure at the focal region. 


\section{Summary and Conclusions}

In this paper, basic fluid dynamics features of the shock focusing process in water inside logarithmic spiral structures are discovered through singleshot and high-speed photography. Quantitative measurement of the pressure distributions is also obtained through BOS technique. The peak pressure along the center line can be compared with the numerical simulations. Complex fluid dynamics such as cavitation occur both in front and behind the shock wave in water. Cavitation behind the shock wave is due to bubble nucleation sites in the water-filled region that get excited when the shock waves passes them. More complex fluid dynamics models would be necessary in the future in order to correctly model the cavitation bubble events and possible pitting and interaction with the surrounding solid surfaces.

Computer simulations are also conducted using the Overture package. The two dimensional rigid boundary simulation confirms that the shock wave undergoes a focusing process, and reaches a peak pressure at the focal region of the logarithmic spiral shape. The fluid-structure simulation, which includes the material effects from the surrounding boundary, indicates that the coupling between fluid and structure changes the shock wave dynamics. As a result, the peak pressure magnitude at the focal region is lower in the two dimensional FSI simulation as compared to the rigid boundary simulation. By contrast, a rigid boundary axisymmetric simulation demonstrates a much higher peak pressure when compared to the two dimensional rigid boundary case, indicating a three-dimensional simulation would be necessary to infer the correct response due to three-dimensional effects.

When comparing the experiments with the simulations, the overall dy- 
namics of the shock front agrees well. The experimental reconstructed peak pressure from BOS is an order of magnitude lower than in the two-dimensional rigid boundary simulation if the incident shock Mach number is set to $M_{s}=$ 1.1 but only about three times lower if $M_{s}=1.03$. For FSI simulations with an aluminum material the simulation with $M_{s}=1.03$ is lower than the rigid simulation, but still higher than the experiment. This is attributed to the PMMA confinement used in the experiment, which has a lower acoustic impedance than aluminum and thus more energy will transmit to the surrounding structure. It shows clearly that initial conditions and focusing effects have a large influence on the thermodynamic properties at the focal region. There is also an uncertainty associated with the results obtained using BOS because the spatial averaging with a fixed size interrogation window smears the shock wave over several pixels. In addition, there will be error generated during the digital image correlation and numerical solver calculation process. Ideally, to reduce the error from BOS, a camera with faster frame rates and higher resolution is needed. Another reason that accounts for the differences observed between the experiments and simulations is the estimation of shock strength by using Hugoniot data taken from literature. If exact material properties of each component used in the experiments were readily available, this should provide more accurate data to use for shock Hugoniot matching techniques.

The main conclusions are summarized below:

1. Shock focusing in water can efficiently be obtained using a logarithmic spiral geometry. Results show that the focusing process at the design Mach number $\left(M_{s}=1.1\right)$ results in up to 13.4 times higher peak pres- 
sure than the lower Mach number $\left(M_{s}=1.03\right)$.

2. The surrounding material influences the shock focusing in several ways:

(a) A rigid material, with a high impedance mismatch, will allow less waves to transmit through the surrounding structure and thus the pressure inside the water-filled domain is higher. For our experiments, the impedance of aluminum, PMMA, and polycarbonate is $17.2 \pm 0.32 \times 10^{6} \mathrm{Ns} / \mathrm{m}^{3}, 2.7 \pm 0.14 \times 10^{6} \mathrm{Ns} / \mathrm{m}^{3}$, and $3.4 \pm 0.07 \times 10^{6} \mathrm{Ns} / \mathrm{m}^{3}$ respectively.

(b) The wave speeds in the surrounding material may cause cavitation ahead of the incident shock wave by putting the water in tension.

3. The simulations show that three-dimensional effects are important for realistic applications where planar two-dimensional geometries are nontypical. Providing accurate initial conditions is important, as the simulations performed here show. For example, a $6 \%$ change in initial shock Mach number changes the maximum pressure at the focal region with a factor close to 10.

4. BOS is a non-intrusive technique that allows for density measurements and then pressure can be inferred from a known equation of state. Higher resolution photographs and a higher framing rate would lead to more accurate BOS results.

In the future, the degree of coupling between the surrounding structure and the water-filled region will be investigated in detail. A simulation of a polycarbonate or PMMA surrounding material would provide insight in how much the peak pressure decays due to change in material properties. This is particularly interesting for thin structures with a high degree of fluid- 
structure coupling, and therefore the role of the type of material, material properties and specific geometry has to be understood. Future work also include shock focusing in thin water-filled convergent surrounding structures made of materials with more relevance to marine-type applications, e.g. steel, aluminum and carbon fiber composite. The fluid-structure coupling effect will be compared to the bulk materials presented here, and further investigated to understand the dynamic response of the surrounding structure.

\section{Acknowledgments}

The authors are grateful for support from Office of Naval Research through through a MURI Grant Number N00014-06-1-0730 (Dr. Y.D.S. Rajapakse, Program Manager). The authors wish to thank Prof. Bill Henshaw at Rensselaer Polytechnic Institute for help with the simulations. We also want to thank the USC Viterbi Machine Shop as well as the Caltech Aero Shop.

Arienti, M., Hung, P., Morano, E., Shepherd, J.E., 2003. A level set approach to Eulerian-Lagrangian coupling. Journal of Computational Physics 185, $213-251$.

Arienti, M., Shepherd, J.E., 2002. Superseismic loading and shock polars: An example of fluid-solid coupling. AIP Conference Proceedings 620, 251-254.

Chaussy, C., Brendel, W., Schmiedt, E., 1980. Extracorporeally induced destruction of kidney stones by shock waves. Lancet 316, 1265-1268.

Chesshire, G., Henshaw, W.D., 1990. Composite overlapping meshes for the solution of partial differential equations. J. Comput. Phys. 90, 1-64. 
Cocchi, J., Saurel, R., Loraud, J., 1996. Treatment of interface problems with godunov-type schemes. Shock Waves 5, 347-357.

Coleman, A.J., Saunders, J.E., 1989. A survey of the acoustic output of commercial extracorporeal shock wave lithotripters. Ultrasound in Medicine \& Biology 15, 213-227.

Doukas, A.G., Kollias, N., 2004. Transdermal drug delivery with a pressure wave. Advanced Drug Delivery Reviews 56, 559-579.

Eliasson, V., Apazidis, N., Tillmark, N., 2007. Controlling the form of strong converging shocks by means of disturbances. Shock Waves 17, 29-42.

Eliasson, V., Henshaw, W., Appelö, D., 2008. On cylindrically converging shock waves shaped by obstacles. Physica D: Nonlinear Phenomena 237, 2203-2209.

Eliasson, V., Mello, M., Rosakis, A., Dimotakis, P., 2010. Experimental investigation of converging shocks in water with various confinement materials. Shock Waves 20, 395-408.

Espinosa, H., Lee, S., Moldovan, N., 2006. A novel fluid structure interaction experiment to investigate deformation of structural elements subjected to impulsive loading. Experimental Mechanics 46, 805-824.

Fleck, N.A., Deshpande, V.S., 2004. The resistance of clamped sandwich beams to shock loading. J. Appl. Mech. 71, 386-401.

Gardner, J.H., Book, D.L., Bernstein, I.B., 1982. Stability of imploding 
shocks in the CCW approximation. Journal of Fluid Mechanics 114, 4158.

Gerald, H., 1997. Use of extracorporeal shock waves in the treatment of pseudarthrosis, tendinopathy and other orthopedic diseases. The Journal of Urology 158, 4-11.

Giordano, J., Jourdan, G., Burtschell, Y., Medale, M., Zeitoun, D.E., Houas, L., 2005. Shock wave impacts on deforming panel, an application of fluidstructure interaction. Shock Waves 14, 103-110.

Guderley, G., 1942. Starke kugelige und zylindrische Verdichtungsstöße in der Nähe des Kugelmittelpunktes bzw. der Zylinderachse. Luftfahrt Forsch. $19,302-312$.

Henshaw, W.D., 2003. OverBlown : A Fluid Flow Solver For Overlapping Grids, Reference Guide, Version 1.0.

Henshaw, W.D., Schwendeman, D.W., 2003. An adaptive numerical scheme for high-speed reactive flow on overlapping grids. Journal of Computational Physics 191, $420-447$.

Henshaw, W.D., Schwendeman, D.W., 2006. Moving overlapping grids with adaptive mesh refinement for high-speed reactive and non-reactive flow. Journal of Computational Physics 216, $744-779$.

Inoue, O., Imuta, S., Milton, B., Takayama, K., 1995. Computational study of shock wave focusing in a log-spiral duct. Shock Waves 5, 183-188. 
Inoue, O., Takahashi, N., Takayama, K., 1993. Shock wave focusing in a log-spiral duct. AIAA J 31, 1150-1152.

Johnsen, E., Colonius, T., 2008. Shock-induced collapse of a gas bubble in shockwave lithotripsy. J. Acoust. Soc. Am. 124, 2011-2020.

Kato, M., Ioritani, N., Suzuki, T., Kambe, M., Inaba, Y., Watanabe, R., Sasano, H., Orikasa, S., 2000. Mechanism of anti-tumor effect of combination of bleomycin and shock waves. Cancer Science 91, 1065-1072.

Lindl, J.D., McCrory, R.L., Campbell, E.M., 1992. Progress toward ignition and burn propagation in inertial confinement fusion. Phys. Today 45, $32-$ 40.

Meier, G., 2002. Computerized background-oriented schlieren. Experiments in Fluids 33, 181-187.

Milton, B., Archer, R., 1969. Generation of implosions by area change in a shock tube. AIAA J 7, 779-780.

Mulley, A.G., 1986. Shock-wave lithotripsy. N Engl J Med 314, 845-847.

Perry, R.W., Kantrowitz, A., 1951. The production and stability of converging shock waves. J. Appl. Phys. 22, 878-886.

Ridah, S., 1988. Shock waves in water. Journal of Applied Physics 64, $152-158$.

Schwendeman, D., Whitham, D., 1987. On converging shock waves. Proc. R. Soc. Lond. A413, 297-311. 
Shin, Y.S., 2004. Ship shock modeling and simulation for far-field underwater explosion. Computers \& Structures 82, 2211 - 2219.

Sturtevant, B., Kulkarny, V.A., 1976. The focusing of weak shock waves. Journal of Fluid Mechanics 73, 651-671.

Takayama, K., Kleine, H., Grönig, H., 1987. An experimental investigation of the stability of converging cylindrical shock waves in air. Exp. Fluids 5, 315-322.

Wagner, W., Pruß, A., 1995. The IAPWS formulation 1995 for the thermodynamic properties of ordinary water substance for general and scientific use. Journal of Physical and Chemical Reference Data 31, 387-535.

Wang, C., Eliasson, V., 2012. Shock wave focusing in water inside convergent structures. International Journal of Multiphysics 6, 267-281.

Wang, C., Qiu, S., Eliasson, V., 2013. Quantitative pressure measurement of shock waves in water using a schlieren-based visualization technique. Experimental Techniques, Article first published online: DOI: 10.1111/ext.12068.

Weiland, D., Lee, C., Ugarte, R., Monga, M., 2007. Impact of shockwave coupling on efficacy of extracorporeal shockwave lithotripsy. Journal of Endourology 21, 137-140.

Whitham, G., 1974. Linear and nonlinear waves. Wiley-Interscience.

Xi, X., Zhong, P., 2001. Dynamic photoelastic study of the transient stress 
${ }_{643}$ field in solids during shock wave lithotripsy. The Journal of the Acoustical 644 Society of America 109, 1226-1239.

${ }_{645}$ Young, Y.L., Liu, Z., Xie, W., 2009. Fluid-structure and shock-bubble in646 teraction effects during underwater explosions near composite structures. 647 Journal of Applied Mechanics 76, p. 051303. 